\title{
Normativité et intentionnalité : pour un pluralisme normatif
}

Par MARIE-HÉLÈne DESMEULES

Université Concordia, Montréal

Résumé Brentano et Husserl reconnurent tous deux que certaines de nos intentionnalités posent des enjeux normatifs. Seulement, Brentano croyait que les intentionnalités ne peuvent être qualifiées normativement que si elles sont incompatibles avec une autre façon opposée de se rapporter intentionnellement à un même objet. Husserl insista pour sa part sur le fait qu'il n'y a des enjeux normatifs que là où il $\mathrm{y}$ a une relation intentionnelle et positionnelle. Malgré cette distinction, en imposant ces conditions (incompatibilité et position) pour qu'il soit question de normativité intentionnelle, Brentano et Husserl se trouvèrent tous deux à favoriser un réductionnisme normatif. Je proposerai pour ma part, dans un geste rappelant celui de la pragmatique d'Austin mais sans m'y restreindre pour autant, de dépasser ce réductionnisme normatif en cessant de limiter à ces conditions l'application de normes à nos intentionnalités.

Depuis sa réactualisation par Brentano, le thème de l'intentionnalité fut repris par plusieurs auteurs pour décrire la spécificité des vécus de la conscience par rapport aux phénomènes physiques. Ces reprises présentent une apparence d'unité puisque, reprenant un seul et même terme maintenant devenu canonique, elles semblent s'entendre sur ce qui constitue le propre des phénomènes psychiques : le fait d'être intentionnel. Pourtant, différentes lignes de fractures creusèrent cette tradition, et les reprises de ce terme ne furent pas toutes fidèles aux enseignements de Brentano ${ }^{1}$. Ce fut le cas de

${ }^{1}$ Parmi les autres fractures, pensons à la définition pratique de l'intentionnalité
adoptée par Maurice Merleau-Ponty et plus récemment par Iris Marion Young, ou 
Husserl qui s'éloigna de son maître en adoptant notamment une définition normative de l'intentionnalité ${ }^{1}$. Selon cette définition, ce qui caractériserait en propre les intentionnalités, c'est de pouvoir être jugées normativement. Là où Brentano reconnaissait encore que certaines intentionnalités ne posent pas d'enjeux normatifs, Husserl croyait pour sa part que l'intentionnalité était spécifiquement de l'ordre de ce à quoi nous pouvons appliquer des normes.

Malgré cette distinction, Brentano et Husserl favorisèrent tous deux, et chacun à sa façon, un réductionnisme normatif. Chez Brentano, la normativité intentionnelle se réduisait toujours à une question d'incompatibilité entre deux phénomènes psychiques opposés. Chez Husserl, les normes qu'il appliquait aux intentionnalités se réduisaient à un seul modèle normatif. Selon le modèle promu par Husserl, ce qui est posé intentionnellement dans la visée de sens intentionnelle doit être confirmé par le remplissement intuitif. Si tel est le cas, la visée de sens peut être qualifiée de «correcte »; dans le cas contraire, elle doit être qualifiée d'«incorrecte». Ce réductionnisme normatif conduisit même Husserl à prendre l'acte intentionnel de juger comme modèle pour penser toute normativité intentionnelle.

À l'encontre de ces réductionnismes normatifs, je propose d'adopter un pluralisme normatif. Un tel pluralisme normatif revient à dire qu'il y a plusieurs normes qui sont appliquées à nos visées de sens et à nos actes intentionnels. Il serait donc possible par exemple de reconnaître que les intentionnalités pratiques posent des problèmes de rectitude sans assimiler ces problèmes de rectitude à la rectitude qui vaut pour les intentionnalités théoriques, et sans non plus les réduire à la question de savoir s'il y a une

celle existentielle adoptée par Jean-Paul Sartre. Young affirme par exemple : «En tant que pure présence au monde et ouverture à ses propres possibilités, le corps est le siège de l'intentionnalité. L'acte intentionnel primordial est par conséquent le mouvement du corps s'orientant selon son milieu et s'y mouvant. Il n'y a monde pour un sujet que dans la mesure où son corps possède certaines capacités par lesquelles il peut se repérer dans son milieu, s'en saisir et se l'approprier selon son orientation intentionnelle. » (Young, Marion I., «Lancer comme une fille. Une phénoménologie de la motilité, de la spatialité et du comportement corporel féminins », trad. D. Landes, Symposium, vol. 21, no 2 (automne 2017), p. 30). Sur la distance entre la définition brentanienne et husserlienne de l'intentionnalité et celle existentielle de Sartre, voir: FARGES, J., «Intentionnalité et réflexion : Éléments pour une confrontation des phénoménologies sartrienne et husserlienne », Bulletin d'Analyse Phénoménologique, vol. 6 (2010), p. 244-263.

1 Sur le lien entre normativité et intentionnalité chez Brentano et Husserl : $C f$., BENOIST, J., « Un concept normatif de l'intentionalité ?» Études phénoménologiques, vol. 23 (2007), p. 9-36. 
incompatibilité entre divers actes intentionnels. Et ce sans renier par ailleurs, si tant est qu'il soit question d'une normativité intentionnelle, que c'est encore quelque chose de spécifique au caractère intentionnel de l'intentionnalité qui nous justifie d'appliquer toutes ces normes à nos intentionnalités.

Pour ce faire, je commencerai par expliciter le cadre qui empêcha Husserl, mais déjà avant lui Brentano, de reconnaître un tel pluralisme normatif. J'identifierai sous quelles conditions il était possible selon eux de parler de normativité intentionnelle. Brentano croyait qu'il n'y avait des enjeux normatifs que là où il y avait une opposition, une incompatibilité. Husserl, de son côté, identifia deux conditions pour qu'il soit possible de parler de normativité : il devait à ses yeux y avoir une relation de type intentionnelle et celle-ci devait inclure une position. Ces conditions eurent cependant pour effet de restreindre leurs considérations normatives. Je proposerai donc, dans un geste rappelant celui de la pragmatique d'Austin, mais sans m'y restreindre pour autant, de dépasser ce réductionnisme normatif en cessant de limiter à ces conditions l'application de normes à nos intentionnalités.

Avant d'entamer l'examen du lien entre intentionnalité et normativité, j'aimerais ajouter quelques clarifications terminologiques. Lorsqu'une norme est appliquée à quelque chose, elle nous permet de le qualifier de " correct » ou d' « incorrect ». Parler de «norme », ce n'est donc pas parler d'un principe nous permettant de distinguer ce qui est possible et ce qui est impossible. La distinction entre le possible et l'impossible, si elle est ontologique, ne recoupe pas la distinction normative : ce qui est correct, tout comme ce qui est incorrect, peut être possible ou impossible. Et c'est bien tout le problème des enjeux normatifs : ce qui est incorrect est tout à fait possible, et ce qui est correct est parfois impossible. Parler de "norme », c'est également ouvrir un espace où il fait sens de parler de devoir, selon le principe qui veut que ce qui est correct doit être et que ce qui est incorrect ne doit pas être. Mais cela ne veut cependant pas dire que ce devoir s'entende au sens fort et impératif, comme synonyme d'« exiger ${ }^{1}$. Ni qu'il s'entende au sens faible et

\footnotetext{
${ }^{1}$ Husserl lui-même distinguait, dès les Prolégomènes, le devoir qui est synonyme d'exiger et le devoir qui est synonyme de rectitude: HUSSERL, E., Recherches logiques, tome 1 : Prolégomènes à la logique pure [Hua XVIII], trad. H. Élie, A. L. Kelkel et R. Schérer, Paris : Presses universitaires de France (coll. Épiméthée), 2002, p. 43-44 [53].
} 
ontologique, comme synonyme de «fortement probable $»^{1}$. Dire de A qu'il doit être B, cela peut parfois simplement dire qu'il est correct que A soit B. Le normatif a une logique qui lui est propre, qui n'est ni ontologique ni impérative.

\section{Intentionnalité et rectitude chez Brentano}

Aux yeux de Brentano, tous les phénomènes psychiques ont un trait commun : ils sont tous intentionnels. Selon sa définition maintenant devenue canonique :

Ce qui caractérise tout phénomène psychique, c'est ce que les Scolastiques du Moyen Âge ont appelé l'inexistence intentionnelle (ou encore mentale) d'un objet et ce que nous pourrions appeler nous-mêmes — en usant d'expressions qui n'excluent pas toute équivoque verbale - la relation à un contenu, la direction vers un objet (sans qu'il faille entendre par là une réalité (Realität)) ou objectivité (Gegenständlichkeit) immanente ${ }^{2}$.

En chacun d'eux, nous avons conscience de quelque chose : nous sommes dirigés vers un objet dont nous avons conscience.

Ce qui a cependant moins été mis de l'avant, ce sont les différences que Brentano reconnaissait entre ces phénomènes psychiques. Car si chaque phénomène psychique a un objet, et parfois même le même objet, « chacun le contient à sa façon », nous dira Brentano ${ }^{3}$. Il proposa même une classification de ces différentes façons de se rapporter à un objet. Il répartit ainsi, à la suite de Descartes ${ }^{4}$, les phénomènes psychiques en trois classes: la classe des représentations, la classe des jugements, et la classe affectivo-volitive à laquelle appartiennent les sentiments. Par représentation, Brentano entendait l'acte par lequel quelque chose nous apparait, que cet apparaître soit un phénomène physique, comme le son, les odeurs, les arbres et les hommes, ou un phénomène psychique, comme lorsque nous nous représentons un acte

\footnotetext{
${ }^{1}$ Comme lorsque nous disons « il doit neiger à Montréal », au sens de «il est fort probable qu'il neige à Montréal ».

${ }^{2}$ BRentano, Franz, Psychologie du point de vue empirique, trad. M. De Gandillac, Paris: Librairie philosophique J. Vrin (Bibliothèque des textes philosophiques), 2008, p. 101 [124-125].

${ }^{3} I d .$, p. 101 [124-125]. Cf., Id., p. 199 [8-9] ; p. 221 [38].

${ }^{4}$ Brentano, F., L'Origine de la connaissance morale, trad. M. de Launay et J.C. Gens, Paris : Gallimard (coll. Bibliothèque de philosophie), 2003, p. 50-51.
} 
d'imagination, une audition, ou un jugement ${ }^{1}$. Sous la classe des jugements, il rassemblait les actes doxiques tels que croire, supputer, douter, etc., que ceux-ci soient affirmatifs ou négatifs ${ }^{2}$. Le domaine affectif et volitif de la conscience, c'est-à-dire à la fois le plaisir et le déplaisir, la joie et la tristesse, la volonté et le souhait, était pour sa part inclus dans la classe des sentiments ${ }^{3}$.

Je ne retracerai pas chacune des différences que Brentano répertoria entre les phénomènes psychiques et qui le justifièrent de les répartir précisément en trois classes. Ce qui m'intéresse, c'est une dichotomie fondamentale qui scinde sa classification des phénomènes psychiques. La classe des jugements et la classe affectivo-volitive avaient à ses yeux quelque chose en commun que n'avait pas la classe des représentations. Il s'ensuivait qu'il ne faisait pas sens, à ses yeux, de parler de normativité intentionnelle pour les représentations, alors qu'il faisait sens d'en parler pour les jugements et les sentiments. Dans la suite de mon propos, je mettrai au jour cette condition normative qui faisait défaut aux représentations, mais pas aux autres classes intentionnelles. Je montrerai ainsi qu'aux yeux de Brentano, il ne peut y avoir d'enjeux normatifs pour les intentionnalités que là où elles peuvent être corrigées, c'est-à-dire transformées en une autre façon opposée, incompatible, de se rapporter à un même objet ${ }^{4}$.

${ }^{1} C f$. , Brentano, F., Psychologie du point de vue empirique, op. cit., p. 95 [114] ; p. 217 [34] ; BRENTANO, F., L'Origine de la connaissance morale, op. cit., p. 50.

${ }^{2} C f$., BREnTANO, F., L'Origine de la connaissance morale, op. cit., p. 51.

${ }^{3}$ Le chapitre VIII du deuxième livre de la Psychologie du point de vue empirique porte exclusivement sur cette classe de phénomènes psychiques: BRENTANO, F., Psychologie du point de vue empirique, op. cit., p. 251-278 [83-124]. Voir également: BRENTANO, F., L'Origine de la connaissance morale, op. cit., p. 51 ; p. 213.

${ }^{4}$ C'est aussi ce décalage entre les intentionnalités normatives et celles a-normatives que Jocelyn Benoist mit de l'avant dans son article «Un concept normatif de l'intentionalité ?» (BENOIST, J., « Un concept normatif de l'intentionalité ? », loc. cit.). Je déplacerai cependant légèrement les problèmes qui étaient ceux de Jocelyn Benoist dans cet article. Je ne chercherai pas à savoir si une intentionnalité a besoin de pouvoir être sujette à une norme pour être, c'est-à-dire si la normativité fait partie de la définition de l'intentionnalité (Id., p. 10-11), ni s'il faut admettre une intentionnalité à chaque fois qu'il est question de normativité, c'est-à-dire si l'intentionnalité fait partie de la définition de la normativité (Id., p. 20-22). Ces problèmes conduisirent Jocelyn Benoist à conclure que : « $\langle\mathrm{c}\rangle$ hez Brentano, le thème intentionnel n'est donc pas originairement normatif, mais la normativité joue un rôle décisif dans son extension. » (Id., p. 22). Pour ma part, je me demanderai à quelle condition il fait sens aux yeux de Brentano de parler de normativité dans le 


\section{Normativité et reconnaissance [Anerkennung]}

Pour nous convaincre que les représentations sont $a$-normatives aux yeux de Brentano, il nous faut suivre les arguments qui lui permirent de conclure que chacune de nos représentations s'accompagne d'une forme de connaissance, et donc d'une forme de jugement. Cette connaissance était même double à ses yeux. Dans Psychologie du point de vue empirique et contre l'idée qu'il puisse y avoir des phénomènes psychiques inconscients, Brentano affirme ainsi que tous les phénomènes psychiques s'accompagnent d'une connaissance dirigée vers le phénomène psychique lui-même. Si cette thèse ne semblait pas faire problème dans le cas des jugements et des sentiments ${ }^{1}$, il concède cependant alors qu'il n'est pas évident qu'une telle connaissance accompagne aussi les représentations. Les représentations semblent poser problème puisque certaines d'entre elles ne contiennent aucune forme de liaison d'idées, et donc aucune forme de connaissance - si tant est que connaître, c'est juger, c'est-à-dire lier $^{2}$. Prenant alors pour exemples des représentations de phénomènes sensibles, lesquelles sont simples et ne contiennent donc aucune liaison, Brentano propose de tout de même y voir à l'œuvre une forme de connaissance - la reconnaissance [Anerkennung $]^{3}$ redevable à une perception interne elle-même simple et sans liaison :

cas des intentionnalités. Condition que Jocelyn Benoist identifia également dans son article, mais sans s'y attarder (Cf., Id., p. 16).

${ }^{1}$ « Nous pensons, nous désirons quelque chose, et nous savons que nous pensons et désirons. » (Brentano, F., Psychologie du point de vue empirique, op. cit., p. 151 [195]).

${ }^{2}$ Brentano rejeta l'idée que tout acte de juger implique une forme de liaison d'idées. Il croyait que les jugements, tout comme les représentations, peuvent être simples ou complexes. Les représentations telles que «un arbre vert, une montagne dorée, un père de cent enfants, un ami de la science » sont complexes (Id., p. 51). Par ailleurs, Brentano refusait de faire des jugements tels que «Le soleil existe » ou «L'arbre vert est» des jugements complexes parce qu'il ne croyait pas que ce genre de jugement consiste en une liaison entre un sujet et le prédicat « existence ». Il refusait par ailleurs explicitement l'idée selon laquelle un des traits distinctifs entre les représentations et les jugements seraient que les premières sont simples alors que les seconds sont complexes. Sur ces questions : $c f$., BRENTANO, F., Psychologie du point de vue empirique, op. cit., p. 225-237 [44-63].

3 Le terme « reconnaissance »[Anerkennung], tout comme le terme approbation, n'est pas très clair en français. En revanche, sous sa forme verbale, il me semble que nous comprenons mieux ce que voulait dire Brentano : chaque fois que nous avons un acte psychique, nous reconnaissons, c'est-à-dire distinguons et discernons, jusqu'au point de l'admettre, l'acte psychique lui-même. 
Quiconque prend garde à ce qui se passe en lui au moment où il entend ou voit, quiconque perçoit son audition ou sa vision est obligé de se rendre à l'évidence : ce jugement de la perception interne ne consiste pas dans la liaison d'un acte psychique pris comme sujet et de l'existence, prise comme prédicat, mais d'une simple reconnaissance et approbation [Anerkennung] du phénomène représentée dans la conscience interne ${ }^{1}$.

Ainsi, avoir une représentation (ou tout autre acte psychique), c'est toujours en même temps savoir que l'on a cet acte psychique.

Mais ce n'est là qu'une des deux choses que nous pouvons reconnaître dans les actes psychiques. Car selon Brentano, qui développe cette idée dans Psychologie descriptive (1887-1891), une reconnaissance se tourne également vers l'objet dont nous avons conscience dans nos actes psychiques. Or, si cette idée ne pose pas de problème dans le cas des jugements et des sentiments, elle semble problématique dans le cas des représentations. Brentano persiste cependant à affirmer que toute représentation d'un objet s'accompagne en même temps d'une « reconnaissance assertorique aveugle » de cet objet représenté ${ }^{2}$. Ainsi, se représenter un objet, ce serait en même temps savoir que l'on se représente et savoir quel est l'objet que l'on se représente.

Dans les arguments présentés par Brentano pour nous convaincre d'admettre cette seconde forme de reconnaissance dans le cas des représentations, la question de la normativité, c'est-à-dire la question de la rectitude de l'intentionnalité en regard de l'objet qu'elle vise, joue un rôle primordial. Ce qui conduit en effet Brentano à admettre une forme de reconnaissance de l'objet représenté, c'est la possibilité de certaines illusions sensibles. Ce genre d'illusions sensibles démontre à ses yeux que la rectitude d'une représentation peut être problématique, c'est-à-dire qu'elle peut être qualifiée de « vraie» ou de «fausse », et qu'une forme de connaissance devait donc s'y adjoindre.

Cet exemple d'illusion sensible, que Brentano doit à Aristote, est synthétisé de la façon suivante par Arnaud Dewalque :

L'expérience, bien connue, est la suivante : lorsque l'on place le médius audessus de l'index et que l'on touche une bille placée entre les deux doigts, on

\footnotetext{
${ }^{1} I d .$, p. 155 [201].

${ }^{2}$ Brentano, F., Psychologie descriptive, trad. A. Dewalque, Paris : Gallimard (coll. Bibliothèque de philosophie), 2017, p. 179 [86].
} 
croit, en se basant sur les sensations tactiles, sentir deux billes, alors que la vue confirme qu'il n'y en a qu'une'.

Il convient de s'attarder sur cet exemple, car son choix n'est pas anodin. Dans cette illusion, il y a deux représentations - la représentation tactile et celle visuelle. Ces deux représentations ont chacune un objet représenté : la vue représente une bille, alors que le toucher représente deux billes. Or, ces deux objets représentés sont incompatibles : il ne peut pas y avoir deux billes et n'y avoir qu'une bille. La synthèse concordante des sens est ainsi mise en déroute : il n'y a pas de synthèse unitaire possible entre « une bille vue » et «deux billes senties au toucher ». Les deux représentations ne peuvent donc pas être toutes les deux vraies, et il faut tout au moins que l'une des deux soit fausse.

Aux yeux de Brentano, il suffisait de présenter cet exemple pour conclure qu'une reconnaissance de l'objet représenté accompagne toutes nos représentations ${ }^{2}$. Mais avant d'expliquer pourquoi, je dois faire trois remarques sur l'exemple mobilisé par Brentano, et avant lui par Aristote. Car le choix de cet exemple d'illusion était délibéré, et j'irais même jusqu'à dire qu'il n'y a que ce genre d'expérience illusoire - c'est-à-dire un bris de la synthèse concordante des sens - qui pouvait aider Brentano à démontrer qu'il y a une forme de reconnaissance qui accompagne toute représentation. Son exemple m'autorise en effet les remarques suivantes :

I) Dans cet exemple, il y a un conflit entre deux objets représentés, et non pas entre deux façons opposées de se rapporter à un même objet représenté. Nous ne sommes pas en présence d'une représentation affirmative d'un objet représenté $\mathrm{A}$ et d'une représentation négative de ce même objet représenté $\mathrm{A}$, auquel cas nous serions devant deux façons incompatibles de se rapporter à un même objet. La vue et le toucher ne sont

\footnotetext{
${ }^{1}$ Pour cette synthèse d'Arnaud Dewalque : Id., p. 179, note de bas de page 2.

${ }^{2}$ Brentano affirme ce qui suit : «4. La relation à l'objet primaire semble être d'un bout à l'autre une relation double : (1) une représentation, (2) une reconnaissance assertorique aveugle. 5. Ce dernier point pourrait susciter des réserves. Pour qu'il soit correct, il ne suffit pas d'établir maintes fois [qu'une reconnaissance assertorique aveugle accompagne la relation à l'objet primaire], mais il faut que cela soit toujours établi et que [la reconnaissance] s'avère être inséparable [de la relation à l'objet primaire]. Aristote semble être de cet avis. [Il attribue] la vérité [et] la fausseté à la sensation. Dans l'expérience des deux doigts, [l'un des deux] sens maintient l'affirmation, alors même que l'autre la contredit. » (Id., p. 179-180). Même après avoir évalué les contre-arguments présentés par certains détracteurs, c'est encore cet argument, qu'il hérite d'Aristote, qu'il retiendra : Id., p. 182.
} 
pas non plus deux façons opposées de se rapporter au même objet représenté : elles sont simplement deux façons différentes de se rapporter à un objet représenté (par la vue et par le toucher). Au contraire, ce sont les objets que ces deux sens représentent qui sont incompatibles.

II) C'est parce que les deux objets représentés sont incompatibles que l'une des représentations doit être fausse. Brentano ne part pas d'une représentation trompeuse isolée - par exemple la vue du bâton brisé dans le verre d'eau, la vue d'un mirage ou une hallucination - , mais plutôt de deux représentations dont la synthèse est impossible. Brentano conclut ensuite, par un raisonnement, qu'une des deux représentations doit être fausse. Que ce soit à partir de deux objets représentés (et non pas d'un seul) et suite à un raisonnement (et non pas directement, dans sa façon de se confirmer) que l'on puisse conclure que les représentations peuvent être fausses n'est pas anodin. Brentano pensait-il que la représentation isolée ne donne jamais à elle seule d'indices ou d'éléments pour conclure, voire avoir l'expérience directe (par exemple dans son manque de clarté, ou dans son caractère brouillé ou flou par rapport à un optimum), qu'elle peut être fausse ? La question mérite d'être posée, puisqu'elle permet d'insister sur le détour logique pris par Brentano. Ce qui est certain, c'est que dans cet exemple, l'application du prédicat «faux» aux représentations ne va pas de soi : elle résulte d'un raisonnement fondé sur l'incompatibilité de deux représentations que l'on cherchait à synthétiser.

III) L'exemple de Brentano met en scène deux représentations qui subsistent et qui offrent un degré de conviction similaire. Brentano ne décrit pas le passage d'une représentation à une autre, dans lequel une première représentation serait dépassée et corrigée par une seconde, contraire et meilleure. Il ne donne pas l'exemple du passage d'une première représentation, saisissant bien deux billes du bout de deux doigts, à une seconde représentation, beaucoup plus optimale, saisissant bel et bien une bille au bout de cinq doigts. Ni celui d'une accommodation visuelle, dans laquelle partant d'une vision floue représentant deux billes, nous passons à une vision nette saisissant une seule bille. Nous sommes devant deux représentations qui subsistent même si l'une d'elles doit être fausse, tout comme dans les apparences empiriques décrites par Kant : même si je sais que la mer à l'horizon décrit une courbe descendante parce que la terre est sphérique, il n'empêche que je vois sa ligne d'horizon comme étant plus haute que le rivage $^{1}$. Ce qui veut également dire, si nous devions reprendre la distinction

${ }^{1}$ KANT, E., Critique de la raison pure, trad. A. Renaut, Paris : Flammarion (coll. Garnier Flammarion), 2006, p. 331 [A 296/B 352]. Brentano fait référence à 
kantienne entre l'illusion et la faute, que nous sommes en présence d'une illusion qui ne disparaît pas même si l'on sait qu'elle est trompeuse (et non pas en présence d'une faute qui pourrait être corrigée). Ce que confirme d'ailleurs Brentano, lorsqu'il affirme que s'il y a une correction, ce n'est pas au niveau des représentations, qui elles persistent, mais par l'intervention d'actes supérieurs de l'ordre du jugement, qui discriminent quelle représentation nous devrions suivre ${ }^{1}$.

On comprend donc pourquoi Brentano qualifie alors la reconnaissance de l'objet représenté d' ' aveugle » : la représentation à elle seule ne permet ni de déceler l'erreur (il en faut deux), ni d'être corrigée (c'est le jugement qui la corrige $)^{2}$. Surtout, ces remarques nous aideront à comprendre pourquoi Brentano pensait nous convaincre qu'une croyance accompagne toute représentation simplement en nous montrant qu'un objet représenté peut être qualifié de « faux».

Nous pourrions répondre à cette question en disant simplement que la fausseté et la vérité sont des prédicats qui appartiennent à la sphère du jugement. Dire d'un objet représenté qu'il peut être dit « vrai » ou «faux », ce serait donc dire qu'il doit d'une façon ou d'une autre être question de jugement. Mais cette réponse n'est guère suffisante.

Pour répondre à cette question, il nous faut plutôt retourner à une opposition que l'on trouve au niveau des jugements et des sentiments, mais qui est absente des représentations ${ }^{3}$. Aux yeux de Brentano, les actes

Helmholtz et non pas à Kant lorsqu'il traite de la persistance des illusions d'optique, mais l'idée reste la même : « Nombreux sont ceux (y compris Helmholtz) qui disent que les illusions d'optique continuent à exister alors même que l'on [s'est] aperçu de l'erreur (peu importe que le penchant instinctif à croire soit fondé ou bien donné par l'habitude). L'intuition claire [Einsicht] prédominera naturellement chez ceux qui sont rationnels. Ils agiront en conséquence. »(BRENTANO, F., Psychologie descriptive, op. cit., p. 180-181 [87])

${ }^{1}$ Id., p. 181 [87-88].

${ }^{2}$ Il semble également que Brentano la qualifie d'aveugle afin de la distinguer de la reconnaissance qui se tourne vers l'acte psychique lui-même. Cette dernière reconnaissance est une évidence immédiate, qui permet de connaître avec le plus haut degré de conviction ce que sont les actes psychiques et la conscience (BRENTANO, F., Psychologie du point de vue empirique, op. cit., p. 156 [202-203]). C'est d'ailleurs ce qui justifie le primat de la psychologie sur les autres sciences de la nature : $I d$., p. 3239 [28-38]. Non pas qu'il ne fasse pas sens de parler de normativité intentionnelle dans le cas de la reconnaissance tournée vers les actes psychiques, mais plutôt que la normativité intentionnelle n'est pas un problème pour cette reconnaissance.

${ }^{3} I d$., p. 239 [65-66]. 
psychiques appartenant aux classes des jugements et des sentiments peuvent se rapporter de deux façons opposées aux objets vers lesquels ils se dirigent. Un jugement peut ainsi accepter ou rejeter un objet, c'est-à-dire l'affirmer ou le nier, alors que le sentiment peut par exemple l'aimer ou le haïr'. Et bien qu'elles s'opposent, ces façons de se diriger vers l'objet — c'est-à-dire aimer et haïr, tout comme affirmer et nier - peuvent se rapporter à un même objet.

Or, ce n'est pas le cas des représentations selon Brentano. Si deux objets représentés peuvent certes être opposés, il n'y a pas deux façons opposées de se représenter le même objet :

Entre les représentations, nous ne trouvons pas de contraires, hors des objets qui s'y trouvent appréhendés. En tant que la chaleur et le froid, la lumière et l'obscurité, un son aigu et un son grave, etc., constituent de tels contraires, nous pouvons dire que la représentation de l'un s'oppose à celle de l'autre. Et dans un autre sens, il n'existe absolument aucune opposition dans le domaine entier de ses activités psychiques. Du fait que l'amour et la haine s'y ajoutent, il se manifeste une espèce toute différente de contrariété. L'opposition ici n'est pas conflit d'objets, car le même objet peut être aimé ou haï; c'est une opposition entre les relations du sujet à l'objet ${ }^{2}$.

De façon similaire, dans la Doctrine du jugement correct, Brentano prendra l'exemple de la représentation du blanc et de la représentation du noir : ces deux représentations sont une relation à deux objets " opposés », mais elles ne sont pas deux façons contraires de se représenter un même objet ${ }^{3}$. Par ailleurs, dans l'exemple d'illusion empirique que j'ai commenté, c'était les objets représentés «une bille » et « deux billes » qui étaient opposés, et non pas deux façons distinctes de se représenter un même objet. Et s'il en était ainsi, c'était simplement parce qu'il n'y a pas, dans le cas de la représentation, d'opposition entre deux façons de se représenter un même objet.

Or, c'est ici, me semble-t-il, que se situe le cœur de l'argument de Brentano lorsqu'il cherche à nous convaincre qu'il y a une forme de reconnaissance qui s'adjoint à chacune de nos représentations. Souvenons-nous comment Brentano était parvenu à la conclusion que les objets représentés

\footnotetext{
${ }^{1}$ Brentano, F., L'Origine de la connaissance morale, op. cit., p. 51 [17-8].

${ }^{2}$ Brentano, F., Psychologie du point de vue empirique, op. cit., p. 239 [65-66].

3 Brentano soutient, dans la Doctrine du jugement correct, que : «<d $>$ ans les représentations, il n'y a pas d'oppositions en dehors de celles qui sont relatives aux objets : clair-sombre, sonore-silencieux, chaud-froid, etc. Parmi les jugements, il y a en revanche une opposition dans la relation à l'objet. » (BRENTANO, F., «La Doctrine du jugement correct », op. cit., p. 214 [33])
} 
peuvent être «faux» : c'était à partir de l'incompatibilité de deux objets représentés. Logiquement, cette incompatibilité signifiait que tout au moins un des deux objets représentés - et peut-être même les deux — devait être qualifié de «faux». Il en concluait également qu'une forme de reconnaissance devait accompagner les représentations. Pourquoi ? Parce que pour appliquer les prédicats «vrai » et «faux», «correct» et «incorrect», à l'acte psychique lui-même, il faut que cet acte psychique s'oppose à un autre acte psychique qui lui est contraire. Dans sa conférence «L'Origine de la connaissance morale », après avoir souligné qu'il y a une opposition dans les jugements et les sentiments, mais pas dans les représentations, Brentano dit en effet :

Ce qui entraîne cette conséquence non négligeable: aucune des activités rangées dans la première classe <celle des représentations> ne peut être qualifiée de juste ou d'erronée; en revanche, pour ce qui est de la deuxième classe <celle des jugements>, et dans chaque cas, des deux modes de relation, acceptation et rejet, l'un sera juste, l'autre erroné, comme le fait valoir, depuis toujours, la logique. Et il en va naturellement de même pour la troisième classe aussi. Des deux modes d'attitude opposés, amour et haine, plaisir et déplaisir, dans chaque cas, l'un est juste - et seulement l'un des deux —, l'autre, erroné'.

Et cela se comprend aisément. Même si l'objet représenté devait en toute logique se révéler «faux», nous ne pouvons pas nous le représenter autrement que nous nous le représentons. Nous ne pouvons encore et toujours que nous le représenter d'une seule et même façon : en nous le représentant. La correction n'est donc pas possible au niveau des représentations, car il n'y a pas de façon opposée, "vraie », de nous représenter un même objet «faux ». Ainsi, comme je l'ai dit dans ma troisième remarque sur l'illusion sensible utilisée par Brentano, le phénomène représentatif subsiste tel qu'il est même si l'on sait que l'objet représenté est faux. Et s'il subsiste, c'est qu'il ne peut pas être autrement qu'il est. Il est une illusion au sens kantien, et non pas une faute, justement parce qu'il ne peut pas être corrigé.

La seule façon de parler d'un phénomène psychique qui serait incorrect dans le cas de l'objet représenté faux, ce serait donc d'adjoindre à l'acte représentatif un autre phénomène psychique pour lequel il fait sens de parler de faute. Cet autre phénomène psychique qui peut être corrigé en une façon opposée de se diriger vers le même objet représenté, c'est la reconnaissance

${ }^{1}$ BREnTANO, F., L'Origine de la connaissance morale, op. cit., p. 52-53. 
assertorique aveugle. La reconnaissance, c'est-à-dire une forme de croyance, de jugement minimal, parait en effet idéale pour remplir cette fonction. Non seulement elle n'exige même pas de liaison d'idées — et concorde ainsi avec les représentations simples -, mais elle peut également être corrigée par un autre jugement de niveau supérieur se rapportant de façon opposée, et donc «vraie », à un même objet représenté : en le niant ${ }^{1}$. En ce sens, la reconnaissance fait partie des phénomènes psychiques pour lesquels il fait sens de parler de « faute ».

Chez Brentano, ce qui est primordial pour qu'il puisse être question de normativité, c'est donc l'incompatibilité. Pourquoi ? Déjà parce que, suivant le passage que j'ai commenté de Psychologie descriptive, c'est l'incompatibilité des objets représentés qui nous permet d'y introduire l'idée de fausseté au niveau des représentations. Pourtant, les représentations ne peuvent pas elles-mêmes, en tant que phénomènes psychiques, être qualifiées normativement. Il ne peut être question de normativité intentionnelle qu'au niveau des jugements et des sentiments. Et encore une fois, c'est l'incompatibilité qui est au cœur de cet autre argument: si un rapport à l'objet ne s'oppose pas à une autre façon incompatible de se rapporter au même objet, ce rapport à l'objet ne peut pas être dans un rapport ayant le caractère de la correction. Car advenant que ce rapport soit «faux », il n'y a pas de rapport opposé, et donc «vrai », par lequel le corriger. Une telle correction est en revanche possible pour les jugements et les sentiments ; eux seuls ont des couples de façons opposées de se rapporter à un même objet.

\section{Les représentations et leur perfection}

Il y a tout au moins une objection qui pourrait m'être adressée. Brentano ne reconnaissait-il pas, dans Psychologie du point de vue empirique, une perfection propre aux représentations ? Celles-ci ne pourraient-elles donc pas être dites plus ou moins correctes, selon qu'elles sont plus ou moins parfaites? Reconnaître des enjeux de perfection propres aux représentations, n'est-ce pas leur reconnaître des enjeux de rectitude?

\footnotetext{
${ }^{1}$ Il est vrai que Brentano admettait une seule forme de reconnaissance à ce niveau : la reconnaissance assertorique et aveugle. En revanche, comme elle est une forme de jugement, elle peut très bien être corrigée par un autre jugement à un niveau supérieur. La représentation ne peut en revanche être corrigée par une autre représentation, puisqu'il n'y a pas de façon opposée de se représenter le même objet.
} 
Pour répondre à cette objection, je propose de faire un retour au chapitre VII du second livre de Psychologie du point de vue empirique. Au $\S 9$, Brentano affirme que les jugements et les actes affectivo-volitifs se distinguent des représentations notamment parce qu'il est possible de leur appliquer des prédicats relatifs à leur perfection et leur imperfection. Il serait possible de qualifier les jugements de «parfait» ou d' «imparfait» selon qu'ils sont vrais ou faux, tout comme il serait possible de qualifier les sentiments de «parfaits» ou d'《imparfaits » selon qu'ils sont bons ou mauvais :

Le domaine de l'amour et de la haine nous révèle donc un mode absolument nouveau de perfection [Vollkommenheit] et d'imperfection [Unvollkommenheit], dont on ne trouve pas la moindre trace dans le domaine des représentations. Du fait que l'amour et la haine s'ajoutent aux phénomènes de représentation, le bien et le mal moral entrent dans le champ de l'activité psychique, du moins le plus souvent et s'il s'agit d'êtres psychiques responsables.

Il en va de même pour le jugement. L'autre espèce de perfection et d'imperfection, tout aussi nouvelle et tout aussi importante, et qui fait complètement défaut, nous l'avons dit, à la pure et simple représentation, appartient en propre au domaine du jugement tout comme celle dont on vient de parler appartient en propre au domaine de l'amour et de la haine. De même que l'amour et la haine sont vertu ou perversité, l'acceptation et la négation sont affirmation vraie ou erreur ${ }^{1}$.

Brentano ne désigne cependant aucun qualificatif de «perfection» ou d' « imperfection » propre aux représentations, et si celles-ci sont dites parfaites ou imparfaites, c'est simplement par « homonymie »:

Dans les représentations, on ne trouve ni vertu, ni perversité morale, ni connaissance vraie, ni erreur. Tout cela leur est intrinsèquement étranger et nous pouvons tout au plus, par homonymie, dire d'une représentation qu'elle est moralement bonne ou mauvaise, vraie ou fausse ${ }^{2}$.

Pourtant, ce silence sur la sorte de perfection propre à la représentation est étonnant, surtout lorsque l'on sait que Brentano clôt le chapitre VIII de ce second livre de Psychologie du point de vue empirique en reconnaissant une forme distincte de perfection à chacune des classes des actes psychiques. Brentano affirme alors que la perfection du jugement est tournée vers les

${ }^{1}$ Brentano, F., Psychologie du point de vue empirique, op. cit., p. 240 [67].

${ }^{2} I d .$, p. 240 [66-67]. 
« vérités qui nous révèlent plus que d'autres une riche plénitude de l'être ", alors que la perfection des sentiments consiste à «pratiquer la vertu et l'amour du bien pour eux-mêmes et dans la mesure de leur perfection $»^{1}$. Or, dans ce dernier passage, la représentation aussi est qualifiée par Brentano de plus ou moins parfaite, selon qu'elle s'approche ou non de l'idéal qui lui est propre : l'idée de Beau. Pourquoi alors avoir d'abord passé sous silence cette perfection propre à la représentation au chapitre VII ?

Nous pourrions répondre en disant qu'au chapitre VII, Brentano voulait seulement éviter que les termes applicables aux jugements et aux sentiments soient appliqués par homonymie aux représentations. Pour ce faire, il n'avait donc pas besoin d'expliciter le type de perfection propre aux représentations. Mais pourquoi alors avoir insisté seulement sur l'homonymie menaçant les représentations? Ne pouvons-nous pas dire également de façon homonyme que la connaissance et la vertu sont belles ?

Je crois donc, et je reconnais que cette thèse est beaucoup plus forte, que ce silence est plutôt dû au fait que Brentano considérait que les perfections qui sont propres aux jugements et aux sentiments présentent des traits similaires que n'a pas la perfection propre à la représentation justifiant ainsi leur mise à l'écart. C'est en effet seulement l'objet qui est plus ou moins parfait dans la représentation, alors que dans le jugement et le sentiment, c'est l'objet et le phénomène psychique grâce auquel il nous est conscient qui sont plus ou moins parfaits. Décrivant succinctement chacune des perfections, Brentano affirme que ce qui constitue la plus haute perfection de la représentation, c'est la contemplation [Betrachtung] du beau ${ }^{2}$. La plus haute perfection du jugement réside pour sa part dans « la connaissance $<\ldots$ de la $>$ riche plénitude de l'être $»^{3}$. Quant à la perfection des sentiments, elle consiste en l'«amour du bien $»^{4}$. Dans tous les cas, une certaine normativité est bien appliquée à l'objet intentionnel : il doit être beau et non pas laid, vrai et non pas faux, bien et non pas mauvais. Mais dans le cas du jugement et du sentiment, il y a en plus une norme appliquée au phénomène psychique lui-même. En parlant de connaissance de la riche plénitude de l'être, Brentano pense à la connaissance positive qui est un jugement affirmatif, par opposition et exclusion du jugement négatif ${ }^{5}$. En parlant de

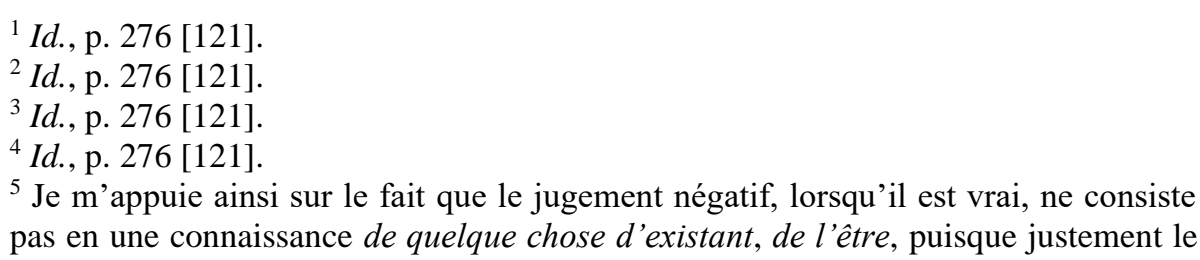


l'amour du bien, il exclut le sentiment de la haine, fût-elle une haine du mauvais ${ }^{1}$. Le jugement négatif et la haine ne pourront jamais prétendre à la perfection du jugement affirmatif et de l'amour. Or, il n'y a pas de norme appliquée à l'acte représentatif lui-même. Brentano dit simplement qu'il faut se représenter, contempler [betrachten], le beau. Mais il ne peut pas alors exclure une autre façon de se représenter le beau, car il n'y a pas, rappelonsnous, d'opposition dans la façon de se représenter un objet, fût-il le beau. $\mathrm{La}$ représentation du beau ne s'oppose qu'à la représentation d'autre chose que le beau — par exemple le laid — ou qu'à l'absence de représentation.

Ce qui veut dire qu'il n'y a pas de norme de perfection appliquée au phénomène intentionnel lui-même au niveau des représentations, mais qu'il y en a une appliquée aux phénomènes intentionnels qui sont de l'ordre du jugement et du sentiment. Ce qui veut aussi surtout dire que le caractère intentionnel de la conscience ne justifie pas à lui seul la possibilité de la qualifier normativement. Ce n'est pas la directionalité de la conscience, le fait qu'elle soit dirigée sur un objet, qui l'ouvre à des qualifications normatives, mais plutôt le fait qu'en se dirigeant sur des objets, elle puisse, pour certaines classes de phénomènes psychiques, le faire de plusieurs façons opposées et incompatibles.

\section{Directionalité, positionalité et rectitude chez Husserl}

Husserl hérita de la réactualisation, par Brentano, du thème de l'intentionnalité pour penser les actes de la conscience. En revanche, les quelques modifications qu'il proposa à cette notion eurent pour effet d'unir plus étroitement ce qu'il entendait par « intentionnalité » et «normativité ». Sa façon de définir l'intentionnalité impliquait qu'il était constitutif des intentionnalités qu'elles conduisent à des problèmes de rectitude. En ce sens, il adopta presque une définition normative de l'intentionnalité. Et si je dis « presque », c'est qu'il ajouta toutefois une condition pour qu'il soit possible

jugement négatif vrai implique que ce qu'il nie n'existe pas : BRENTANO, F., « Le concept de vérité », dans L'Origine de la connaissance morale, op. cit., p. 109-113.

${ }^{1}$ De même, dans "L'Origine de la connaissance morale », Brentano affirme que l'activité du sentiment doit elle-même être bonne. Il remarque alors que la souffrance et la tristesse — et nous pourrions ajouter la haine — , même si elles sont correctes, sont inférieures au plaisir et à la joie — et nous pourrions ajouter l'amour — qui sont corrects : BRENTANO, F., L'Origine de la connaissance morale, op. cit., p. 65 ; p. 66 note $31 ;$ p. 72 note 36 . 
de considérer normativement une intentionnalité : elle devait inclure une position $[\text { Setzung }]^{1}$.

Plusieurs voies peuvent nous conduire à ce constat. Nous pourrions montrer que Husserl ne croyait pas que ce que Brentano nommait représentation était une intentionnalité ${ }^{2}$. Comme la représentation était la seule intentionnalité $a$-normative chez Brentano, Husserl, en n'y reconnaissant pas une intentionnalité, se retrouvait ainsi seulement avec des intentionnalités normatives. Cependant, cette voie pose quelques problèmes : comme nous le verrons, certaines des intentionnalités que Husserl reconnaissait et auxquelles il appliquait des prédicats normatifs sont à classer parmi ce que Brentano considérait appartenir à la classe des représentations. Une seconde voie, déjà empruntée par nombre de commentateurs ${ }^{3}$, consiste à montrer que la norma-

1 Il est également question de position dans l'article « Un concept normatif de l'intentionalité ?» de Jocelyn Benoist. Il montre alors que la position est, aux paragraphes 37 et 38 de la Recherche logique $\mathrm{V}$, une condition pour qu'il soit question d'actes objectivants : «C'est ce parallélisme, et ce seul parallélisme, qui fonde la notion rigoureuse d'acte objectivant. C'est-à-dire que, concrètement, la notion d'《acte objectivant» renvoie à celle d'un acte par lequel on peut poser quelque chose ou non (donc qui connaît une dimension positionnelle). » (BENOIST, J., « Un concept normatif de l'intentionalité ? », loc. cit., p. 25). En 1901, le problème des actes évaluatifs serait donc, aux yeux de Husserl, que ceux-ci n'incluent aucune position. Or, la suite du propos de Jocelyn Benoist consiste à montrer que si en 1908 Husserl réussit à reconnaître certains vécus affectivo-volitifs comme des intentionnalités, c'est grâce à un « déplacement essentiel » (Id., p. 27). Husserl aurait cessé d'y chercher une position (ces vécus ne posant pas l'être des objets), et se serait plutôt concentré sur la dimension normative de ces vécus $(I d .$, p. 30 ; p. 32 ; p. 34). Mon propos prendra en revanche appui sur un texte (l'Exkurs à l'Introduction à l'éthique) dans lequel Husserl procède de façon exactement inverse. Comme nous le verrons, c'est en insistant d'abord sur la positionalité des actes affectivo-volitifs que Husserl conclut qu'ils sont aussi sujets à des prédicats normatifs.

${ }^{2}$ Gyemant, M., «Repräsentation et intentionnalité : Sur l'impossibilité de purger l'intentionnalité de tout objet immanent», Bulletin d'analyse phénoménologique, vol. 6 (2010), p. 29-45.

${ }^{3}$ Sur cet enjeu, voir : PRADELlE, D., «Une problématique univocité de la raison », préface de Leçons pour l'éthique et la théorie de la valeur, Paris: Presses universitaires de France (coll. Épiméthée), 2009, p. 47-52 ; PRADELLE, D., Par-delà la révolution copernicienne, Paris: Presses universitaires de France (coll. Épiméthée), 2012, p. 228-235 ; BENOIST, J., « Un concept normatif de l'intentionalité ? », loc. cit., p. 9-36; MARIANI, E., « L'éthique à l'épreuve de la raison. Critique, système et méthode dans les Vorlesungen über Ethik und Wertlehre (1908-1914) de E. Husserl » dans Feeling and Value, Willing and Action. Essays in the Context of a 
tivité était un trait intentionnel si important aux yeux de Husserl que pour accepter de parler d' « intentionnalité » pour les actes appartenant à la classe affectivo-volitive, il chercha s'il était possible de parler pour ces actes de correction et de rectitude au même sens que pour les actes judicatifs. N'eût été la possibilité d'appliquer des normes aux actes affectivo-volitifs similaires à celles appliquées aux actes appartenant à la sphère du jugement, Husserl aurait donc refusé de parler d'actes intentionnels pour les vécus affectivo-volitifs. Pour ma part, je proposerai une troisième voie, qui me permettra de mettre de l'avant l'Exkurs in der Vorlesung, texte écrit entre 1920 et 1924 et peu connu ${ }^{1}$. Dans ce texte, Husserl prit pour point de départ la structure de l'intentionnalité afin de montrer qu'en raison du type de relation qui subsiste entre le sens intentionnel et l'objet réel auquel il se rapporte, il fait sens d'appliquer au sens intentionnel des prédicats normatifs. C'est donc en considérant d'abord le sens intentionnel, et non pas l'opposition qui subsiste entre différentes façons de se rapporter à un même objet (voie qui était celle de Brentano), que Husserl lia intentionnalité et normativité. Et plus spécifiquement, ce serait la position [Setzung] se trouvant au sein du sens intentionnel qui nous justifierait à ses yeux d'appliquer au sens et aux actes intentionnels des prédicats normatifs.

\section{Normativité, directionalité et position}

Les considérations normatives de Husserl dans l'Exkurs à ses Leçons sur l'éthique de 1920-1924 prennent d'abord appui sur une classification des sciences qu'il propose ${ }^{2}$. Il remarque que certaines sciences considèrent leurs objets objectivement, c'est-à-dire qu'elles ont pour thème ce que sont leurs objets de recherche, quelles sont leurs propriétés, et quelles relations subsistent entre eux. D'autres en revanche considèrent leurs objets normativement, de sorte que des prédicats tels que « correct» et «incorrect», «vrai» et « faux », « authentique » et « inauthentique », voire des propositions ayant la forme « $\mathrm{x}$ doit être $\mathrm{ou}$ « $\mathrm{x}$ ne doit pas être », interviennent.

Phenomenological Psychology, éd. Ubiali, M. et Wehrle, M., Cham : Springer, (coll. Phaenomenologica), 2015, p. 18-24.

${ }^{1}$ HUSSERL, E., Einleitung in die Ethik. Vorlesungen Sommersemester 1920 und 1924 [Hua XXXVII], éd. H. Peucker. Dordrecht: Kluwer Academic Publishers, 2004, [259-320].

${ }_{2}^{2} I d$., [259-264]. 
Ce que cherche alors Husserl dans cet Exkurs, c'est ce qui fonde la possibilité de cette orientation normative des sciences. Il répond à ce problème en faisant un retour à la structure intentionnelle de la conscience, et principalement à la relation entre le sens intentionnel et l'objet auquel il se rapporte. L'intentionnalité aurait une structure précise, celle d'une imbrication entre le moi, son acte, le sens et l'objet :

Par principe, le moi, son acte, le sens et l'objet nous sont donnés seulement dans une imbrication [Ineinander] inextricable ; nous ne pouvons pas penser un moi sans le penser en train de réaliser des actes ou réalisant possiblement des actes; les actes ne peuvent pas être pensés sans qu'ils visent quelque chose, et donc sans qu'ils aient un sens; nous ne pouvons pas penser le sens sans une objectivité qui est justement visée dans ce sens ; et c'est pourquoi le moi et les actes sont en même temps rapportés à cette objectivité ${ }^{1}$.

Si le moi se rapporte intentionnellement à des objets dans des actes, il le ferait donc en les visant par du sens. Or, pour Husserl, c'est à cette inclusion du sens dans la structure intentionnelle que nous devons la possibilité d'appliquer des prédicats normatifs à certains éléments de cette structure.

Pour nous en convaincre, Husserl décide d'insister sur ce que la relation entre le sens et l'objet auquel il se rapporte aurait de spécifique ${ }^{2}$. Il commence d'abord par remarquer que cette relation n'est pas un rapport d'inclusion réelle. Prenant l'exemple de la planète Terre (et aussi de la planète Mars), Husserl affirme que la Terre réelle à laquelle se réfère la proposition «La Terre est ronde» n'est pas réellement dans le sens intentionnel - si elle est «réellement» quelque part, c'est dans l'espace réel. Il ajoute ensuite que la relation entre le sens et l'objet auquel il se rapporte n'est pas non plus un rapport entre un tout et ses parties. La planète Terre n'est pas une partie de la proposition « La Terre est ronde ». En effet, il n'y a que ce qui appartient à l'ordre du sens qui peut être une partie d'une proposition. Or, la Terre, en tant que planète réelle, n'est pas de l'ordre du sens. Prétendre le contraire, c'est commettre un contresens, c'est-à-dire aller à l'encontre des relations aprioriques matérielles et réunir deux éléments qui ne vont pas ensemble, qui n'ont pas rapport ensemble, bref, deux éléments un élément appartenant au domaine du réel et un élément appartenant au domaine du sens — qui n'appartiennent pas au même domaine d'objet.

Husserl est alors peu loquace sur les raisons qui le conduisent à insister sur l'exclusion de ces deux types de rapports pour comprendre la relation

${ }^{1} I d .,[262]$.

${ }^{2} I d$., [261-262]. 
entre le sens intentionnel et l'objet auquel il se rapporte. Pour comprendre pourquoi il commence par exclure ces deux rapports, il faut cependant nous rappeler que ce que souhaite Husserl dans l'Exkurs, c'est de trouver ce qui fonde la distinction entre les sciences objectives, c'est-à-dire ontologiques, et les sciences normatives. Or, les deux types de rapports exclus par Husserl (l'inclusion réelle et le rapport entre le tout et les parties) sont tous deux des relations ontologiques, c'est-à-dire des relations qui subsistent entre deux entités qui existent et parce qu'elles existent — que ce soit réellement ou idéalement. Le but de Husserl en excluant d'emblée ces deux rapports, c'est donc de montrer que le sens intentionnel et l'objet auquel il se rapporte sont dans une relation qui n'est justement pas de l'ordre de celles qui valent dans les sciences objectives, c'est-à-dire dans une relation qui n'est pas ontologique.

C'est pourquoi, me semble-t-il, après avoir exclu ces deux rapports, il nous donne l'exemple d'une visée de sens qui ne peut justement pas être dans une relation ontologique avec l'objet auquel elle se rapporte parce que l'objet auquel elle se rapporte n'existe pas. Cet exemple, c'est celui d'une proposition portant sur le dieu Fitzlijutzli : même s'il y a bel et bien un sens qui se rapporte au dieu Fitzlijutzli, dieu qui n'existe pas, il n'en demeure pas moins que le sens est en relation à un objet ${ }^{1}$.

Quel genre de relation peut-il alors y avoir entre quelque chose qui est (le sens) et quelque chose qui n'est pas (l'objet auquel il se rapporte) ? Cette relation ne peut pas être ontologique, puisqu'un des deux termes peut ne pas exister (dans la relation ontologique, lorsqu'un des termes fait défaut, il n'y a pas de relation ontologique). Pourtant, il y a bien une relation entre le sens et l'objet, que celui-ci soit ou non réellement. L'objet, qu'il existe ou non, est visé par le sens, et c'est cette visée qui les met en relation: le sens vise l'objet et l'objet est visé grâce au sens. Ils sont donc dans une relation de visée, une relation intentionnelle.

C'est à ce genre de relation intentionnelle entre le sens et l'objet auquel il se rapporte que Husserl impute l'apparition de prédicats normatifs. En effet, il nous est toujours possible de qualifier normativement l'un des termes de ce genre de relation en prenant l'autre terme de la relation comme norme. C'est-à-dire que c'est précisément parce que dans ce genre de relation l'un des termes, c'est-à-dire l'objet auquel se rapporte le sens, peut faire défaut qu'il fait sens d'y voir un enjeu normatif. L'enjeu normatif consiste alors à qualifier de «correct », de «vrai », le sens qui vise bel et bien un objet tel qu'il est réellement, et d'incorrect, de «faux », le sens auquel un tel

${ }^{1} I d .,[263-264]$. 
objet fait défaut. Autrement dit, le sens et l'objet peuvent être dans une relation normative dans laquelle c'est le sens qui est sujet à des prédicats normatifs (correct/incorrect, vrai/faux) et où c'est l'objet qui est la norme qui préside à cette qualification.

Nous comprenons donc maintenant pourquoi, dans son fil argumentaire, Husserl tenait à ce point à ce que la relation entre le sens et l'objet ne soit pas une relation ontologique. Si c'eût été le cas, il n'y aurait tout simplement pas eu de relation entre le sens et l'objet en l'absence d'un des deux termes, alors que le but de Husserl, en pensant une relation normative, c'était justement de penser une relation qui subsiste même là où (et surtout parce que) l'un des termes peut faire défaut.

Husserl en conclut que les sciences ayant le sens comme domaine d'objet peuvent avoir deux orientations distinctes. Elles peuvent considérer le sens de façon objective, auquel cas il s'agit de spécifier ce que chaque sens est, quelles sont ses propriétés, quelles sont les formes des unités de sens et des enchaînements de propositions ${ }^{1}$, etc. Les sciences portant sur le sens peuvent également le considérer normativement, auquel cas il s'agit d'établir quelles sont les connexions qui subsistent entre le sens, d'une part, et les prédicats normatifs, d'autre part ${ }^{2}$.

Pourtant, bien que ce genre de relation intentionnelle soit nécessaire pour qu'il fasse sens d'appliquer des prédicats normatifs au sens, elle n'est pas suffisante. Husserl admettait en effet qu'il y a des intentionnalités qui sont $a$-normatives, auxquelles il ne fait aucun sens d'appliquer des prédicats normatifs. C'est le cas par exemple des actes intentionnels qui ont été neutralisés, où on ne fait que penser des contenus de sens sans poser l'être ou l'être-ainsi (par exemple l'être-rond de la Terre) d'un objet. Penser de façon neutralisée, c'est par exemple penser «La Terre est plate», mais sans

${ }^{1}$ Id., [267]. Parmi les sciences qui portent sur le sens et qui le considèrent objectivement, Husserl range la grammaire pure et la logique pure.

${ }^{2}$ C'est sous cette orientation normative que se place par exemple la logique normative. Des propositions telles que «toute proposition grammaticalement bien formée peut être vraie ou fausse », «si les prémisses "tous les A sont B" et "C est A" sont vraies, alors la conclusion "C est B" est nécessairement vraie» ou «les propositions grammaticalement incomplètes ne sont ni vraies, ni fausses » sont normatives. Elles impliquent que le sens et ses structures sont en connexion avec les prédicats « vrai » et «faux ». Mais il n'est pas besoin de s'élever à la formalité et à la généralité de la logique pour retrouver une telle orientation normative. Attribuer à une proposition les prédicats «vrai» et «faux», comme dans la proposition normative «la proposition "la Terre est plate" est fausse », c'est déjà adopter une orientation normative à l'égard du sens.

21

Bull. anal. phén. XIV 2 (2018)

http://popups.ulg.ac.be/1782-2041/ @ 2018 ULiège BAP 
chercher par là à poser que la Terre est plate'. Dans ce genre d'actes neutralisés, toute thèse d'être a été mise en suspens : la «position » [Setzung] n'a plus d'efficace. Il s'ensuit, comme nous dit Husserl dans les Idées directrices, que pour ce genre d'actes, il ne fait aucun sens de qualifier leur sens intentionnel de vrai ou de faux, de correct ou d'incorrect :

Toute chose caractérisée quant au noème comme étant (comme certaine), comme possible, conjecturable, problématique, nulle, etc., peut être ainsi caractérisée de façon «valable» ou «non valable»; elle peut être, être possible, être nulle, etc., « en vérité ». Par contre on ne "pose » rien tant qu'on se figure simplement par la pensée; ce n'est pas là une conscience positionnelle. La «simple pensée» de réalité, de possibilités, etc., ne "prétend» rien; on n'a ni à la reconnaitre comme correcte, ni à la rejeter comme incorrecte ${ }^{2}$.

Dans ces actes neutralisés, il y a pourtant bien une visée de sens. Il faut donc spécifier ce qui, dans la relation intentionnelle, permet de la qualifier normativement : pour être sujette à une qualification normative, la visée de sens doit inclure une position [Setzung] de l'être ou de l'être-ainsi de l'objet auquel elle se rapporte. C'est cette position qui institue le rapport normatif.

Husserl réitère cette idée dans l'Exkurs, mais cette fois sans faire référence aux actes neutralisés. Dans ce texte, le problème de Husserl est de savoir s'il n'y a que les propositions [Sätze], c'est-à-dire les unités de sens ayant la forme «A est B », attribuant l'être-B de A, qui peuvent être qualifiées normativement. En effet, les prédicats «vrai » et «faux » ne semblent applicables qu'aux visées de sens ayant la forme d'une proposition et donc corrélatives à l'acte de juger. Mais qu'en est-il des visées de sens, comme celles de la perception ou de la représentation, qui n'incluent pas

${ }^{1}$ HUSSERL, E., Idées directrices pour une phénoménologie, tome 1 : Introduction générale à la phénoménologie pure (1913) [Hua III], trad. P. Ricœur, Paris : Gallimard, 1950, p. 367 [222] Ce genre de suspension de la position, de neutralisation, survient par exemple lorsque nous cherchons à analyser grammaticalement la proposition «La Terre est plate »: ce qui nous importe dans ce genre d'analyse grammaticale, c'est la structure grammaticale de la visée de sens, et non pas d'affirmer, ni de vérifier, que la Terre est bel et bien ainsi. De même, la phénoménologie, qui s'intéresse aux vécus de conscience, fait usage de la neutralisation. Pour considérer les vécus de conscience en eux-mêmes, et non pas pour ce qu'ils affirment ou nient des objets auxquels ils se rapportent, elle suspend les positions qu'ils pourraient contenir.

${ }^{2}$ Husserl, E., Idées directrices I, op. cit., p. 369 [223]. 
cette forme propositionnelle ? Qui prennent simplement la forme «A est » ou «A existe »? Peuvent-elles être qualifiées normativement?

Husserl répond à cette question en se demandant s'il y a une position dans ce genre d'actes intentionnels. Le geste de Husserl consiste donc à insister sur le fait que c'est le caractère positionnel, et non la forme propositionnelle, de nos visées de sens qui nous justifie de leur appliquer des prédicats normatifs. Or, ce qu'il remarque, c'est qu'il fait sens de parler de «position» pour les vécus qui se rapporte directement, dans un rayon unique nous dit alors Husserl, à leurs objets, c'est-à-dire sans chercher à leur attribuer des déterminations ${ }^{1}$. Ces vécus, même s'ils ne posent pas ce que sont ces objets, posent néanmoins l'être de l'objet auquel ils se rapportent. Aux yeux de Husserl, cela signifie que leur contenu de sens peut donc être qualifié normativement. Et cela se comprend aisément. Poser que A est, c'est toujours en même temps soumettre normativement notre visée de sens positionnelle à la confirmation — ou à l'infirmation — intuitive de l'existence de A.

Seulement, pour marquer la différence qui subsiste entre ce genre de visées de sens directes et les visées de sens appartenant à la sphère du jugement — les propositions [Sätze] — Husserl spécifie que dans le cas des premières, les prédicats normatifs qui s'appliquent sont des prédicats apparentés au terme «existence », comme «véritablement» [wirklich] et «ne pas » [nichts], comme «étant-véridiquement» [wahrhaft-seiend] et «n'étant pas » [nicht-seiend], ou encore comme « existant» [existierend] et «n'existant pas »[nicht-existierend] $]^{2}$. L'emploi de ces termes par Husserl me semble cependant malheureux, puisqu'il suscite une équivoque : nous ne devons pas les entendre au sens positionnel, comme des synonymes du mot «être ». Ils doivent être employés au sens normatif et s'appliquer spécifiquement à nos visées de sens. C'est pourquoi il est préférable, me semble-t-il, de les traduire de la façon suivante : «A est vraiment » ou «c'est bien le cas que A est » et «en aucun cas A est» ou «ce n'est pas le cas que A est». C'est le sens « A est » qui est alors qualifié normativement, et non pas l'objet A.

\section{La transposition des prédicats normatifs aux actes intentionnels}

Dans l'Exkurs, Husserl ne limite pas l'attribution de prédicats normatifs qu'au sens intentionnel qui inclut une position. Il suggère en effet au $\S 4$ de

\footnotetext{
${ }^{1}$ HuSSERL, E., Einleitung in die Ethik, op. cit., [274-275].

${ }^{2}$ Id., [276].
} 
transposer aux actes intentionnels eux-mêmes les prédicats normatifs qui s'appliquent d'abord à nos visées de sens. Husserl nous dit :

Évidemment la normation des propositions se transpose maintenant aux actes. Reprenons pour le moment les actes judicatifs. Ceux parmi eux qui sont vrais, c'est-à-dire qui dans des fondations évidentes ou allant vers des évidences laissent voir en eux le caractère idéal de la vérité, se nomment actes corrects. Celui qui juge ainsi juge correctement, ce qu'il juge et énonce expressément est une proposition vraie [...] Les mots «vrai » et «faux » et tous les mots ayant un sens similaire détiennent un sens qui peut être transposé aux actes. Lorsque l'on dit que quelqu'un juge de façon vraie, on veut alors dire à la fois que l'acte de juger peut être dit vrai, mais aussi et à proprement parler qu'il juge quelque chose de vrai ; à l'inverse quelque chose de faux lorsque l'on dit d'un acte de juger qu'il est faux. Nous avons un mot spécifique pour dire cela : «errer», alors que le mot «erreur» est plus approprié pour la proposition ${ }^{1}$.

Cette transposition de la qualification normative aux actes intentionnels serait donc due à la structure intentionnelle : si l'acte intentionnel se rapporte à un objet à travers du sens, et si le sens intentionnel par lequel on s'y rapporte est sujet à des prédicats normatifs, alors l'acte lui-même pourra par transposition être qualifié de correct ou d'incorrect, de conforme ou non à la norme qui est appliquée au sens.

Husserl s'éloigne donc considérablement de la façon dont Brentano justifiait l'application de prédicats normatifs aux actes intentionnels. Pour Brentano, la question de savoir s'il fait sens d'appliquer des prédicats normatifs à un acte intentionnel se résumait à la question de savoir s'il est possible de modifier l'acte en question en une façon opposée de se rapporter au même objet. Pour Husserl, le problème est résolu autrement. S'il fait sens d'appliquer des prédicats normatifs à la visée de sens de l'acte, ce qui est le cas lorsque le sens se rapporte à son objet en posant l'être ou l'être-ainsi de celui-ci, alors il fait sens de transposer les prédicats normatifs qui valent pour la visée de sens à l'acte intentionnel lui-même. Et ce, parce que la visée de sens et l'acte sont imbriqués : c'est toujours à travers une visée de sens qu'un acte se rapporte à son objet. C'est donc médiatement, par le détour du contenu de sens des actes intentionnels et de sa qualification normative, que

${ }^{1} I d$., [271]. Husserl ajoute en note : «Les actes — ici évidemment les activités du moi, les actions se diffusant à partir du moi en tant qu'unités singulières, qui ont elles-mêmes à nouveau leurs noèses. ». 
Husserl conclut qu'il est possible d'appliquer des prédicats normatifs aux actes intentionnels.

Ainsi, la question de savoir si l'acte intentionnel a un acte intentionnel opposé ne joue aucun rôle lorsque Husserl se demande si une perception, un souvenir ou une représentation, bref ce qui chez Brentano aurait appartenu à la classe des «représentations », peut être dit «correct» ou «incorrect». Pour répondre à cette question, Husserl cherche seulement à savoir si ces vécus se rapportent à quelque chose à travers une visée de sens qui inclut une position, une thèse d'être. Si c'est le cas, et Husserl croit que oui ${ }^{1}$, il fait sens d'appliquer des prédicats normatifs à ces visées de sens et, par transposition, à leurs actes intentionnels.

Il en va de même dans l'Exkurs lorsqu'il est question de savoir s'il y a une normativité à l'œuvre dans la sphère des vécus affectivo-volitifs. Au lieu de chercher à savoir s'il y a un couple d'actes (l'amour et la haine, l'évaluation positive et l'évaluation négative) qui se rapportent de façon opposée à un objet commun, Husserl cherche à savoir si ce genre d'actes inclut une visée de sens positionnelle. Certains vécus de cette sphère

${ }^{1}$ Dans l'Exkurs, Husserl est bref quant à la question de savoir si c'est bien par une visée de sens que nous nous rapportons aux objets dans ce genre d'acte, et s'ils incluent bien une position. Il nous parle à une occurrence du sens de la perception [Wahrnehmungssinn] (Id., [267]). Nous pouvons en revanche consulter les Idées directrices, où Husserl distinguait des vécus sensuels ne contenant aucune visée de sens, comme les simples contenus de sensation de couleur, du toucher, sonore, etc., et des actes ayant un contenu sensuel et une visée de sens, comme la perception du rouge en tant que rouge (HuSSERL, E., Idées directrices I, op. cit., p. 288-289 [172]) ; p. 295 [176]) Quant à la position, Husserl dit dans l'Exkurs qu'il y a une position qui accompagne ce genre d'actes puisque c'est ce genre d'acte simple qui permet la position directe, à rayon unique, d'un simple objet: «Dans la sphère de la connaissance, nous trouvons, comme cela va de soi, que les propositions déterminantes les plus primitives, comme "ceci est rouge", contiennent déjà des propositions, des propositions pures, pour ainsi dire à rayon unique [einstrahlige]; de simples positions d'objet comme "ceci", un tout petit mot, qui indique directement un objet, et ne le fait comme on le comprend aisément que grâce au fait qu'il y a une perception, un souvenir, une représentation [Vorstellung] simple et quelconque, dans lesquels l'objet en question est ce qui est posé dans une simple position, dans une simple proposition d'objet. »(HUSSERL, E., Einleitung in die Ethik, op. cit., [275]). De même, dans les Leçons sur l'éthique de 1908, Husserl rappelle que la perception inclut toujours une prise de position [Stellungnahme], une forme de croyance, en l'être de ce qui est perçu : $C f$., HuSSERL, E., Leçons sur l'éthique et la théorie de la valeur (1908-1914) [Hua XXVIII], trad. P. Ducat, P. Lang et C. Lobo, Paris : Presses Universitaires de France (coll. Épiméthée), 2009, p. 429 [338]. 
affectivo-volitive ont bel et bien une visée de sens nous dit alors Husserl, qui parle d'une « effectuation de sens [Sinnesleistungen] de l'affectivité et de la volonté $»^{1}$. De plus, ce sens peut être positionnel. La position peut être simple, à rayon unique, comme c'était le cas avec la perception, mais Husserl parle alors d'évaluation pour la sphère affectivo-volitive. Sur elle peut cependant également s'édifier des positions ayant la forme de la proposition, c'est-à-dire d'une unité de sens attribuant à un objet axiologique ou pratique telle ou telle valeur :

Tout comme il y a une simple [schlichtes] expérience, et que sur elle s'érige un acte déterminant jugeant ce dont nous faisons l'expérience, il y a une simple évaluation de ce dont nous faisons l'expérience, et sur elle s'érige une évaluation déterminante qui s'y réfère. Il en est ainsi par exemple lorsque l'objet présent dans l'acte affectif comme agréable est parcouru selon ses moments agréables et qu'à travers ceux-ci la valeur affective simplement posée est déterminée dans l'acte affectif singulier².

Conséquemment, il ferait sens aux yeux de Husserl d'appliquer des prédicats normatifs aux visées de sens de la sphère affectivo-volitives. Dès lors qu'il est question de poser l'être ou l'être ainsi d'objets axiologiques et pratiques, il fait sens de se demander si cette visée de sens est correcte ou incorrecte, si l'objet visé a bel et bien telle ou telle valeur, s'il est bel et bien un moyen ou un but, et s'il est véritablement tel qu'il est déterminé dans les propositions axiologico-pratiques. Aussi Husserl note-t-il que :

De la même façon que les objets posés dans la simple position d'objet gagnent, en tant que simple sens, leurs prédicats logico-normatifs que sont les prédicats «étant-véridiquement » [wahrhaft-seiend] et «n'étant pas » [nichtseiend], les valeurs de l'objet posées dans de simples positions de valeur et de volonté gagnent aussi, dans des domaines parallèles, leurs propres prédicats

\footnotetext{
${ }^{1}$ HuSSERL, E., Einleitung in die Ethik, op. cit., [279]. Il affirme également que la conscience évaluante et pratique a ses propres propositions, c'est-à-dire ses propres unités de sens ayant la forme «A est B » (Id., [271]). Nous pouvons penser aux propositions « la Terre est belle » ou « la protection de la Terre est un but pratique ». Sur cette visée de sens propre aux actes affectivo-volitifs : $C f$., HuSSERL, E., Idées directrices I, op. cit., §95 [197-199]. De plus, certains vécus de cette sphère ne contiennent aucune visée de sens. Toute comme Husserl reconnaissait des vécus sensuels non dotés de visée de sens, il reconnaît des vécus affectifs, comme les sensations de plaisir et de douleurs, qui n'incluent aucune visée de sens (Id., p. 288 [172]).

${ }^{2}$ Husserl, E., Einleitung in die Ethik, op. cit., [274].
} 
normatifs axiologiques et pratiques. Mais ici des objets de connaissance, à savoir des objets étant véritablement, et bien qu'ils ne se détachent cependant pas expressément dans le langage, font déjà fonction de substrats de l'évaluation et de la volonté : nous avons donc dans les actes d'évaluer et de vouloir des objets de valeur et des objets pratiques concrets et visés qui sont d'abord posés en eux en tant que choses réelles, mais aussi en tant que choses auxquelles s'attachent des caractéristiques axiologiques et pratiques. C'est ainsi que ces choses sont visées, et la question de la vérité consiste alors à savoir, après que l'on se soit assuré que ces choses sont véritablement, si elles sont à présent des valeurs véritables, des biens véritables, de vrais buts, de vrais moyens ${ }^{1}$.

De plus, comme les vécus appartenant à la sphère affectivo-volitive connaissent la même structure intentionnelle que la sphère judicative, les prédicats normatifs qui s'appliquent aux visées de sens axiologiques et pratiques peuvent également être transposés aux actes intentionnels :

Lorsque la considération axiologique [Werthaltung] est telle que la valeur qui y est posée est une valeur authentique, vraie, et une valeur qui peut s'attester de façon appropriée, lorsque un acte volitif est tel que le but qu'il pose et le moyen qu'il pose sont tous les deux corrects, alors nous parlons d'une évaluation correcte, d'une volonté correcte; sinon, d'une évaluation incorrecte, d'une volition incorrecte ${ }^{2}$.

Dans ce texte et comparativement à Brentano, Husserl pense donc la normativité comme étant intimement liée à la relation intentionnelle elle-même : à ses yeux, l'intentionnalité appartient spécifiquement à l'ordre de ce qu'il fait sens de qualifier de correct ou d'incorrect. La relation entre le sens et l'objet auquel il se rapporte n'étant pas une relation ontologique, celle-ci peut prendre une tournure normative, de façon à ce que l'un des termes devienne une norme pour l'autre terme de la relation. En revanche, aux yeux de Husserl, ce qui institue dans la visée de sens une telle normativité, c'est la position qui s'y trouve — ou du moins, lorsqu'elle n'a pas par exemple été suspendue par neutralisation. La position est donc la condition assujettissant nos intentionnalités à des normes. Et si, ultimement, il n'y a pas que les visées de sens qui soient qualifiables normativement, mais également les actes intentionnels, c'est parce que ceux-ci sont intimement imbriqués aux premiers.

${ }^{1} I d .,[277]$.

${ }^{2} I d .,[271]$. 


\section{Intentionnalités et pluralisme normatif}

Les conditions que Brentano et Husserl identifièrent pour qu'il puisse être question d'une normativité appliquée à nos intentionnalités témoignent de leur réductionnisme normatif. Chez Brentano, les intentionnalités pour lesquelles il n'y avait pas de couple de façons opposées de se rapporter à un même objet n'étaient pas sujettes à des normes. Mais c'est donc dire que sa conception de la normativité était très restreinte : la normativité intentionnelle était simplement attachée à l'impossibilité pour la conscience d'un objet et pour la conscience opposée d'un même objet d'être toutes deux correctes. Pour Husserl, dire d'une intentionnalité qu'elle est dirigée normativement, c'est dire que sa visée de sens positionnelle se dirige selon ce qui se confirme dans le remplissement intuitif. C'est la norme qui est appliquée à l'intentionnalité. Pourquoi ? Parce que dans la relation intentionnelle, l'objet auquel se rapporte une visée de sens peut très bien être différent de la façon dont il est visé et il peut même, au contraire des relations ontologiques, faire défaut. Il est donc tout à fait possible de juger normativement nos visées de sens en prenant l'objet auquel elles se rapportent et dont elles posent l'être que cet objet soit une chose naturelle ou réelle, une valeur, une idée, etc. comme norme. Mais n'est-ce pas, encore une fois, une façon très réductrice de penser le lien entre normativité et intentionnalité ? N'y a-t-il pas d'autres normes qui sont appliquées à nos intentionnalités ? C'est l'hypothèse que j'aimerais maintenant brièvement défendre.

Je pourrais, pour répondre à cette question, reprendre les critiques qu'Austin adressa aux analyses traditionnelles du langage. Tout comme l'approche pragmatique permit de critiquer la réduction des actes langagiers à leur seule fonction constative ${ }^{1}$, je pourrais ainsi soulever contre Husserl que nos intentionnalités ne font pas que poser, que constater, l'être ou l'être-ainsi de quelque chose. C'est un des types de visées de sens que nous pouvons avoir, mais certainement pas le seul. Conséquemment, si nous ne faisons pas que de poser l'être ou l'être-ainsi de quelque chose dans nos intentionnalités, peut-être y a-t-il d'autres normes applicables à celles-ci. L'approche pragmatique me permet par ailleurs de soulever une autre critique à l'endroit de Husserl. Pour ce dernier, si des prédicats normatifs sont appliqués aux actes intentionnels, c'est par transposition de prédicats normatifs qui s'appliquent d'abord et avant tout à leur visée de sens. C'est donc médiatement qu'il fait

\footnotetext{
${ }^{1} C f$., RÉCANATI, F., « Du positivisme logique à la philosophie du langage ordinaire : naissance de la pragmatique », dans AUSTIN, J. L., Quand dire, c'est faire, trad. G. Lane, Paris : Éditions du Seuil (coll. Points. Essais), 1970, p. 185-203.
} 
sens de dire qu'un acte intentionnel est « correct» ou « incorrect». Il en va cependant autrement dans l'analyse pragmatique du langage : c'est l'acte luimême qui peut être jugé normativement et dit «correct» ou «incorrect». Certes, il y a plusieurs sortes d'incorrection pour les actes langagiers : ils peuvent échouer parce que l'effet qu'ils devaient accomplir n'a pas lieu (Austin parle alors d'« insuccès ») ou parce qu'ils n'ont pas été faits pour leurs effets (ce qu'Austin nomme «abus ») ${ }^{1}$. Dans le premier cas, la norme appliquée à l'acte est qu'il ait les effets qui lui sont propres, alors que dans le second, c'est que l'acte soit fait de bonne foi ${ }^{2}$. Mais au-delà de cette différence, l'acte est toujours jugé en tant qu'acte, et non pas pour son contenu de sens. La question normative est de savoir si l'acte fait son effet (réussite) et s'il a été fait pour ses effets (abus).

Pour autant, cette orientation normative propre à l'approche pragmatique ne me satisfait pas non plus. Elle conçoit la normativité selon une directionalité, un être-dirigé-sur, similaire à celle de Husserl. L'acte langagier vise, est dirigé sur « quelque chose », et c'est selon la confirmation ou l'infirmation de ce « quelque chose » qu'il sera jugé correct ou incorrect, c'est-à-dire réussi ou non. Seulement, au lieu qu'il soit question de la confirmation de l'être ou de l'être-ainsi d'un objet tel qu'il est visé par la visée intentionnelle, ce sont les effets conventionnellement adjoints à l'acte intentionnel qui servent de norme à la rectitude — à la réussite — de l'acte langagier ${ }^{3}$. Nous sommes donc encore en présence d'une relation « intentionnelle » similaire à celle de Husserl : l'acte langagier qui vise certains effets peut être présent même si les effets font défaut. Et je ne veux pas dire par là que tout acte langagier est constatif. Viser des effets n'est pas la même chose que viser ce qu'est quelque chose : la première relation intentionnelle est «pragmatique », alors que la seconde est « objective». En revanche, dans les deux cas, il y a bien une relation de visée dans laquelle ce qui est visé et qui

\footnotetext{
${ }^{1}$ Austin, J. L., Quand dire, c'est faire, op. cit., p. 47-56 [12-24].

${ }^{2}$ Nous laissons de côté le sixième cas soulevé par Austin, celui où les personnes ne se comportent pas conformément à l'acte «par la suite» (Id., p. 49 [15]). En effet, il nous semble dans ce cas que ce n'est pas l'acte langagier lui-même qui est incorrect, mais les actes futurs qui ne s'y conforment pas.

${ }^{3}$ Certes, Austin reconnaît plusieurs conditions (existence d'une convention, convenance du contexte, emploi des termes appropriés, personnes appropriées, etc.) qui peuvent faire défaut aux actes, et il semble en ce sens varier ses considérations normatives. En revanche, c'est toujours des conditions pour la réalisation effective des effets que doit avoir l'acte. En ce sens, c'est toujours la même norme qui est ultimement appliquée.
} 
peut pourtant être absent ou différent peut être pris pour norme de la rectitude de la visée.

À ce titre, l'approche pragmatique fait preuve à mon sens d'une pauvreté normative similaire à celle de Husserl. Ce que j'aimerais donc montrer, c'est qu'il n'y a pas que ce qui est visé intentionnellement qui puisse servir de norme appliquée à nos actes et nos visées de sens. Un acte peut être incorrect de bien des façons qui n'ont rien à voir avec l'objet — ou l'effet — qu'il vise. Il peut même être incorrect même s'il vise correctement ce qu'il vise.

Considérons d'abord l'acte de dire un secret. Cet acte peut être incorrect à plusieurs égards. Il est vrai qu'il peut etre incorrect simplement parce que ce qui est divulgué est faux. Il est vrai également que la divulgation peut échouer, ne pas réaliser les effets visés, principalement lorsque ce qui est divulgué était en fait déjà connu. Mais même si (et d'autant plus si) l'acte de divulguer un secret est réussi et même s'il contient tout au moins une part de vérité, l'acte de divulguer un secret peut être jugé incorrect en vertu d'autres normes, comme la fidélité à la parole donnée et la discrétion. Et il y a également des normes qui, en certains contextes, pourraient permettre d'affirmer qu'il est correct de divulguer un secret : si nous prenons par exemple le bien commun comme norme, et que divulguer un secret permet d'éviter un crime. Ce qui est important cependant, c'est de voir qu'aucune de ces normes ne se résume à la question de savoir si ce que l'acte vise est bel et bien tel qu'il l'a visé.

Il en va de même pour l'acte de mentir. Il fait certes sens de qualifier d' « incorrect » l'acte de mentir parce qu'il contient une proposition fausse il serait en ce sens toujours incorrect. Il est aussi possible de qualifier d' « incorrect » l'acte de mentir qui vise à tort une proposition comme étant fausse alors qu'elle est en fait tout à fait vraie. N'est-ce pas là d'ailleurs, dans l'expérience de pensée de Kant, la véritable incorrection commise par celui qui, souhaitant éviter un meurtre, croit mentir alors qu'il dit en fait une proposition vraie ${ }^{1}$ ? L'acte de mentir peut également échouer lorsque, par exemple, les circonstances sont telles que nous ne parvenons pas à

\footnotetext{
${ }^{1}$ Je déplace ainsi le problème de Kant, qui concluait : «C'est donc un commandement de la raison qui est sacré, absolument impératif, qui ne peut être limité par aucune convenance : en toute déclarations, il faut être véridique (loyal). » (KANT, E., Sur un prétendu droit de mentir par humanité, trad. L. Guillermit, Paris : Vrin, 1967, p. 69-70) Mais Kant a-t-il raison de dire que le problème vient du fait que la déclaration n'est pas véridique ? Le problème ne vient-il pas plutôt du fait d'avoir échoué à mentir ?
} 
convaincre autrui de la vérité de notre mensonge. Dans chacun de ces cas, il s'agit de savoir si ce que vise l'acte de mentir, que ce soit l'objet ou l'effet, est bel et bien. Or, l'acte de mentir peut être jugé normativement de bien d'autres façons. C'est le cas, par exemple, lorsque nous lui appliquons la norme de la créance en la parole et concluons qu'il est incorrect. Ou lorsqu'il est qualifié de correct en vertu de la norme de la protection d'autrui. Dans ces cas, la question de la rectitude de cet acte ne se réduit pas à la question de savoir si ce qu'il vise est bel et bien tel qu'il l'a visé.

Il arrive également que nous jugions un acte pour des effets qui ne sont pas spécifiquement visés par lui. C'est le cas par exemple lorsque nous jugeons qu'il est incorrect de remercier quelqu'un par avance. L'effet de l'acte de remercier est d'instituer un rapport avec autrui où nous reconnaissons notre dette envers lui. Mais lorsque nous le remercions par avance, nous réalisons en surplus un autre effet : nous instituons un rapport où l'autre se sent en dette de réaliser ce pour quoi nous reconnaissons notre dette. Pour ne pas que l'être-en-dette de celui qui remercie reste à vide, celui que l'on remercie à l'avance est lui-même mis-en-dette de faire ce dont on le remercie par avance. Remercier par avance, c'est une façon d'obliger quelqu'un, de lier quelqu'un, qui est l'exact inverse du contre-don. Mais c'est aussi pour cet effet périphérique, parce qu'il met en dette celui que l'on remercie, que l'acte de remercier par avance peut être qualifié d'«incorrect». C'est le cas, par exemple, lorsque nous lui appliquons la norme « ne pas contraindre autrui ». Mais ce n'est alors ni son effet visé ni son objet qui est utilisé comme norme.

Je donnerai enfin un dernier exemple : celui des représentations. Brentano et Husserl donnaient une réponse divergente à la question de savoir si une représentation peut être qualifiée normativement. Si Brentano refusait d'y reconnaître une normativité, dans la mesure où il n'est pas possible pour les représentations de se rapporter de différentes façons opposées à un même objet, Husserl acceptait lorsque la représentation, la perception ou le souvenir avait une visée de sens posant l'être de quelque chose. Mais il existe tout au moins une autre norme qui peut être appliquée à ce que Brentano entendait par représentation, et qui fut notamment examinée par Husserl ${ }^{1}$. Cette norme, c'est celle de l'optimalité, c'est-à-dire celle du caractère clair et distinct du contenu de la représentation. Selon cette norme, les représentations dont le contenu permet de distinguer le plus clairement un objet de tout autre objet sont des représentations correctes. Celles dont le contenu pourrait permettre de prendre un objet pour un objet qu'il n'est pas sont qualifiées

${ }^{1}$ Voir notamment : HUSSERL, E., Idées directrices I, op. cit., § 44, p. 83-84.

31

Bull. anal. phén. XIV 2 (2018)

http://popups.ulg.ac.be/1782-2041/ @ 2018 ULiège BAP 
d'incorrectes. C'est le cas par exemple lorsque la luminosité est telle qu'elle fait paraître bleue la neige blanche ; lorsque le surplomb ne me permet de voir que des chapeaux, et non des visages ; ou lorsque la chaleur d'un plat est telle que l'on ne distingue pas toutes les subtilités de ses saveurs. Mais dans chacun de ces cas, il se peut que ce soit notre façon de nous diriger sur ce qui est représenté qui soit jugée normativement. Cette façon sera jugée incorrecte lorsqu'elle ne sera pas «accommodée » à l'optimalité de l'objet représenté : comme lorsque nous sommes trop loin ou trop proche, serrons trop fort ou trop faiblement, faisons le focus visuel trop en avant ou trop en arrière, quand nous sommes dans le mauvais environnement ou utilisons des outils lunette, microscope, éclairage - inadéquats, etc. En ce sens, c'est également notre façon de nous diriger sur ce qui est représenté qui peut être qualifiée de « correcte » ou d' « incorrecte » sous la norme de l'optimalité.

\section{Conclusion}

Ce que ces exemples épars de normes devaient montrer, c'est que nous appliquons plusieurs normes - et le pluriel est important - à nos intentionnalités. Ces normes ne se réduisent pas à la question de savoir si la visée de sens de l'acte intentionnel vise bel et bien l'objet tel qu'il est vraiment, ni à la question de savoir si une façon opposée d'avoir conscience d'un même objet est possible.

Je conclurai en répondant à une objection qui pourrait m'être adressée. Est-ce bien encore à nos vécus en tant qu'ils sont intentionnels que nous appliquons ces normes? Le cœur des propos de Brentano et de Husserl n'était-il pas de chercher les normes qui sont spécifiquement redevables du caractère intentionnel — et positionnel dans le cas de Husserl - de nos vécus ? C'est-à-dire du fait qu'avoir conscience, c'est avoir conscience de quelque chose ? En quel sens est-ce encore le cas pour les normes que j'ai mises de l'avant?

Il ne me semble pas avoir dérogé au cadre intentionnel de Brentano et de Husserl. Seulement, au lieu de commencer par identifier sous quelles conditions il y a une normativité intentionnelle (incompatibilité, position, etc.), j'ai simplement cherché des cas où nous appliquons des normes à des traits essentiels de l'intentionnalité. Depuis Brentano, l'intentionnalité consiste à avoir conscience d'un certain objet et d'une certaine façon. En ce sens, si l'intentionnalité consiste à se diriger sur un certain objet, la norme peut être appliquée au fait que l'acte intentionnel se dirige sur tel objet et non sur tel autre. C'était le cas des intentionnalités qui consistent à exprimer un 
secret ou un mensonge : le problème normatif ne venait pas de l'expression ou de la façon de l'exprimer; il venait de l'objet (dire un secret, dire un mensonge) que l'on choisissait d'exprimer. Et si l'intentionnalité consiste également à se diriger d'une certaine façon sur un objet, la norme peut donc également être appliquée au fait que l'intentionnalité se dirige de telle façon et non pas de telle autre façon. C'était le cas dans mes exemples renvoyant à l'acte de remercier par avance et à l'acte de se représenter de façon non optimale.

Je me permettrai une dernière remarque pour illustrer la révolution normative impliquée par mon propos. Pour Husserl, c'est la relation intentionnelle qui est une relation normative. En effet, l'objet tel qu'il est vraiment est un telos-normatif pour les visées et les actes intentionnels positionnels. Mais il est aussi, même s'il a une telle fonction normative, l'un des termes de la relation intentionnelle - il en est le telos, l' « horizon ». En revanche, dans le pluralisme normatif que j'ai proposé, c'est la relation intentionnelle qui est dans une relation normative envers une norme (l'optimalité, la créance et la fidélité de la parole donnée, etc.). Cette norme lui est donc extérieure. Ce faisant, la norme peut s'appliquer à une partie de la structure intentionnelle ou à l'ensemble de celle-ci. Mais elle le fait toujours sans être elle-même l'un des termes de la relation intentionnelle : elle s'applique sur elle, à la verticale pourrions-nous dire, pour la travailler « du dessus ». 